\title{
User Prioritized Constraint Free Dynamic Bandwidth Allocation Algorithm for EPON Networks
}

\author{
N. Subhashini* and A. Brintha Therese \\ VIT University, Chennai -600127, Tamil Nadu, India; subhashini.n@vit.ac.in, abrinthatherese@vit.ac.in
}

\begin{abstract}
To tackle tremendous growth in the network traffic, EPON (Ethernet PON Networks) based on Time Division Multiplexing (TDM) is being used. There is a need for a Dynamic Bandwidth Allocation (DBA) algorithm to control the flow of sequence of data among the subscribers. In this paper a new user prioritized Dynamic Bandwidth Allocation (DBA) algorithm is demonstrated. Depending on the prioritized request, bandwidth is allocated to each Optical Network Unit (ONU). The paper addresses Inter and Intra ONU allocation. The bandwidth is allocated fairly among the ONUs. The proposed algorithm is evaluated against various dynamic bandwidth allocation algorithms on different performance characteristics like fairness, bandwidth utilization and packet delay using MATLAB. Findings: The simulated results of the proposed algorithm show improvements in performance characteristics compared to the other algorithms.
\end{abstract}

Keywords: Bandwidth Utilization, Dynamic Bandwidth Allocation (DBA), EPON, Fairness, User Prioritization

\section{Introduction}

Today network traffic is tremendously increased due to different applications. To meet the demands arising from various applications, low loss and low-cost equipment is necessary. Ethernet Passive Optical Network [EPON] can be employed for different broadband services as it is less expensive, scalable and can be used with other networks ${ }^{1}$. It uses low cost passive optical components for transmission of packets. It is termed passive since it doesn't employ any active elements in the path of transmission.

EPON basically comprises of Optical Line Terminal (OLT) which acts as a point of access to Wide Area Network (WAN) or Metropolitan Area Network (MAN) on the network side. On the client side the network is equipped with Optical Network Units (ONU). The OLT and ONUs are interconnected by passive optical components. It uses tree topology and Ethernet frames are used for data transmission between them ${ }^{2}$.

Multi-Point Control Protocol (MPCP) is employed for the control information interchange in EPON which involves two MPCP messages; REPORT and GATE. The queue length from each ONU is informed periodically to the OLT by REPORT message. The transmission duration for each ONU calculated by the OLT is notified by the GATE messages. The calculated time duration is known as grant cycle which is separate for each ONU ${ }^{3}$. The transmission of data from OLT to ONUs is termed as downstream transmission while reverse transmission is termed as upstream transmission. In downstream transmission, the packets are broadcasted to each ONU through a passive splitter. The ONUs identifies their relevant information through the identifier code sent along with the data. For upstream transmission, the OLT assigns different time slots for each ONU to prevent collision and loss of data during transmission ${ }^{4}$.

The paper discusses a Dynamic Bandwidth Allocation algorithm which is based on the request from each user. The paper also addresses the implementation of the proposed algorithm. The proposed algorithm is evaluated against existing algorithms and results are presented.

${ }^{*}$ Author for correspondence 


\section{Critical Review}

The main element of the EPON architecture is the algorithm which allocates the bandwidth and grants window size for each ONU. The DBA is crucial for preventing the collisions in the upstream transmission and for making use of bandwidth available to the maximum extent.

The allocation of bandwidth dynamically is grouped into two methods: single-level scheduler and hierarchical scheduler. The algorithm addressed in the paper ${ }^{5,6}$ makes use of single level scheduler and the allotment of bandwidth is done in a round-robin fashion. This algorithm makes ONUs to starve for longer time as it deals with one ONU at a time. This can be overcome by the algorithms proposed in the papers ${ }^{7,8}$ which uses hierarchical scheduler. Hierarchical scheduler differentiates services into three classes thereby allocating different queues for different classes. It is done in two steps: Inter ONU allotment and Intra ONU allotment. In Inter ONU allotment scheme, bandwidth is allocated to each ONU by the OLT where as in Intra ONU allotment scheme, the bandwidth allotted to a particular ONU is divided between different queues of each ONU. But even these algorithms cannot make use of excessive bandwidth from lightly loaded ONUs and allots bandwidth only to a limit set by the algorithm.

The prominent hierarchical scheduling algorithms till date are Min DBA and RDM DBA. Min proposed a DBA algorithm ${ }^{9}$ based on fixed round trip time. This uses limited IPACT for Inter ONU allocation. However, to avoid light load punishment in the lower level scheduler i.e., for Intra ONU allocation, a limitation is set for each type of traffic. The disadvantage of this algorithm is that limitation does not allow the OLT to allocate the bandwidth according to the request from the ONU. The recent algorithm is based on the Russian Doll Model ${ }^{10}$. It uses the available bandwidth to a considerable extent. The proposed algorithm makes use of RDM DBA for the Inter ONU allocation.

The proposed User Prioritized Constraint Free (UPCF) Dynamic Bandwidth Allocation algorithm makes use of bandwidth which is excess in the lightly loaded ONUs and allocates it to the ONUs which are loaded heavily. To overcome the light load punishment in IPACT algorithm, a solution is given in the paper ${ }^{11}$ by making use of the excessive bandwidth. The above algorithm is enhanced by the scheme proposed in the paper $^{12}$. The proposed UPCF algorithm overcomes every problem mentioned above. The proposed UPCF algorithm enhances the bandwidth utilization factor by utilizing surplus bandwidth. It also has a better fairness value as it allots bandwidth fairly to each ONU. The delay which is a major concern of today's world is less compared to the existing algorithms.

\section{Proposed UPCF Algorithm}

The proposed UPCF algorithm is explained in this section. It employs TDM technique to grant the time slots to each active ONUs in the network. The ONU classifies the packet request from the user into three classes (voice, video and data) and identified packets are placed in their respective queues. Through REPORT message, ONUs request the time slot needed for its transmission. The length of the queue for each class from each ONU is determined by the OLT using a high-level scheduler. The request from each ONU is calculated and the available bandwidth is shared among different queues which are also determined by the high-level scheduler. The same is informed to the ONUs through GATE messages. The proposed algorithm allocates the bandwidth among the ONUs (Inter ONU allocation) and again in each ONU (Intra ONU allocation).

\subsection{Inter ONU Allocation}

The proposed algorithm first allocates the bandwidth to each ONU in the OLT. The entire bandwidth is utilized to allocate the requested bandwidth from each ONU. The inter ONU bandwidth allocation based on the $\mathrm{RDM}^{10}$ is explained in the following steps:

1. The total bandwidth requested by each $\mathrm{ONU}$ is computed.

$$
\mathrm{R}_{\mathrm{i}}=\mathrm{R}_{\mathrm{i}}^{\text {voice }}+\mathrm{R}_{\mathrm{i}}^{\text {video }}+\mathrm{R}_{\mathrm{i}}^{\text {data }}
$$

Where $R_{i}$ is the total requested Bandwidth from each ONU, $R_{i}^{\text {voice }}, R_{i}^{\text {video }}, R_{i}^{\text {data }}$ are the requested bandwidths for voice, video and data respectively.

2. A minimum bandwidth $\mathrm{G}_{\min }$ is allocated to the $\mathrm{ONU}$ if the request is null to enable its request during next slot.

$$
\mathrm{B}_{\mathrm{i}}=\mathrm{G}_{\min } \text { if } \mathrm{R}_{\mathrm{i}}=0
$$

Where $\mathrm{B}_{\mathrm{i}}$ is the Allocated Bandwidth. 
3. The requested bandwidth is compared with Gmax i.e., the initial maximum bandwidth that can be allocated to each ONU and the bandwidth allocation is done according to the formula.

$$
B_{i}=\left\{\begin{array}{cc}
\mathrm{R}_{\mathrm{i}}, & \mathrm{R}_{\mathrm{i}} \geq \mathrm{G}_{\text {max }} \\
\mathrm{G}_{\text {max }} & \mathrm{R}_{\mathrm{i}}<\mathrm{G}_{\text {max }}
\end{array}\right.
$$

4. The surplus bandwidth, Bexcess is computed from the lightly loaded ONUs.

$$
\mathrm{B}_{\text {excess }}=\sum \mathrm{G}_{\max }-\mathrm{R}_{\mathrm{i}}(\mathrm{l})
$$

Where $\mathrm{R}_{\mathrm{i}}(\mathrm{l})$ is the requested bandwidth of lightly loaded ONU.

5. The demand bandwidth, Bdemand is computed.

$$
\mathrm{B}_{\text {demand }}=\sum \mathrm{R}_{\mathrm{i}}(\mathrm{k})-\mathrm{G}_{\max }
$$

Where $\mathrm{R}_{\mathrm{i}}(\mathrm{k})$ is the requested bandwidth of heavily loaded ONU,

6. If the surplus bandwidth is greater than the demanded bandwidth, the requested is allocated.

$$
\mathrm{B}_{\mathrm{i}}=\mathrm{R}_{\mathrm{i}}
$$

7. If the surplus bandwidth is lesser than the demanded bandwidth, then it is shared among the high demanded ONUs.

$$
\mathrm{B}_{\text {excess }}(\mathrm{i})=\mathrm{B}_{\text {excess }} \mathrm{x}\left[\mathrm{R}_{\mathrm{i}} / \sum \mathrm{R}(\mathrm{k})\right]
$$

8. Bexcess (i) and Bi are summed up and allocated to each ONU.

$$
\mathrm{B}_{\mathrm{i}}=\mathrm{B}_{\mathrm{i}}+\mathrm{B}_{\text {excess }}(\mathrm{i})
$$

The flow chart for the Inter ONU Bandwidth allocation is shown in the Figure 1.

\subsection{Intra ONU Allocation}

After allocating the bandwidth to each ONU, the algorithm starts apportioning the requested bandwidth

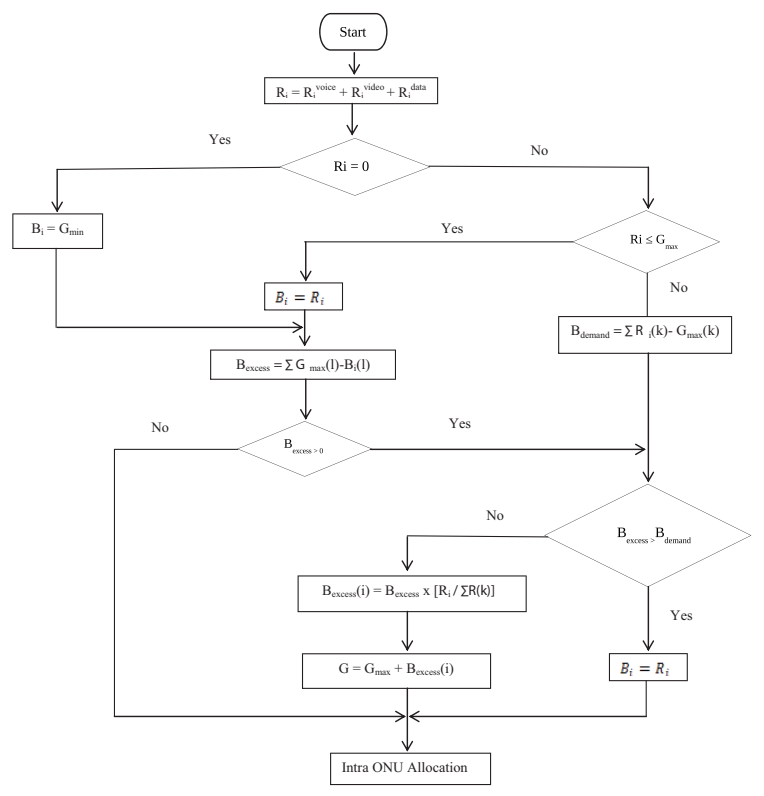

Figure 1. Inter ONU. Allocation

inside ONU depending on the bandwidth available. The request of any particular user would be highest for one's prioritized traffic class and as the UPCF algorithm allocates the bandwidth proportionately, more amount of bandwidth is allocated to the prioritized traffic class and hence it is known as User Prioritized model. The prioritization of the users may vary for each cycle. Unlike other existing algorithms, limitations are not set for Intra ONU allocation and hence it is termed as constraint free model. As the bandwidth is being divided proportionately in the UPCF model, there is no problem of the entire bandwidth being allocated to a single traffic class and from the results it is clear that UPCF model shows better performance.

If the requested bandwidth and the allocated bandwidth are equal then the requested bandwidth to each class is allocated. The total bandwidth available to the $\mathrm{ONU}$ is shared among the three classes depending on the request. Here, voice is not given the preference as in the earlier algorithms since the proposed algorithm is based on user priority. The intra ONU allocation is as follows:

$$
\begin{gathered}
\mathrm{B}_{\mathrm{i}}^{\text {voice }}=\mathrm{R}_{\mathrm{i}}^{\text {voice }} \\
\mathrm{B}_{\mathrm{i}}^{\text {video }}=\mathrm{R}_{\mathrm{i}}^{\text {video }} \\
\mathrm{B}_{\mathrm{i}}^{\text {data }}=\mathrm{R}_{\mathrm{i}}^{\text {data }} \\
\mathrm{B}_{\mathrm{i}}^{\text {voice }}, \mathrm{B}_{\mathrm{i}}^{\text {video }} \text { and } \mathrm{B}_{\mathrm{i}}^{\text {data }}
\end{gathered}
$$

are the bandwidths allocated to voice, video and data traffics respectively. 
If the requested bandwidth is greater than the allocated bandwidth then the bandwidth divided proportionally among the three classes based on their request:

$$
\begin{aligned}
& B_{i}^{\text {voice }}=B_{i}\left[\frac{R_{i}^{\text {voice }}}{R_{i}^{\text {voice }}+R_{i}^{\text {video }}+R_{i}^{\text {data }}}\right] \\
& B_{i}^{\text {video }}=B_{i}\left[\frac{R_{i}^{\text {video }}}{R_{i}^{\text {voice }}+R_{i}^{\text {video }}+R_{i}^{\text {data }}}\right] \\
& B_{i}^{\text {data }}=B_{i}\left[\frac{R_{i}^{\text {data }}}{R_{i}^{\text {voice }}+R_{i}^{\text {video }}+R_{i}^{\text {data }}}\right]
\end{aligned}
$$

\section{Results}

The UPCF algorithm is implemented and simulated using MATLAB. The simulation parameters considered are given in Table 1. The algorithm is evaluated on the performance characteristics like bandwidth utilization, packet delay and fairness index to show that UPCF algorithm provides better QoS. The comparative study is made on the existing DBA algorithms like Min DBA9 and RDM DBA10. These algorithms are employed for comparative study since they both use hierarchical scheduling for inter and intra ONU allocation with three traffic classes. The three algorithms are tested under similar simulation parameters.

Table 1. Simulation parameters

\begin{tabular}{|c|c|c|}
\hline Symbol & Description & 1GB EPON \\
\hline $\mathrm{N}$ & $\begin{array}{c}\text { Total numbers of Optical } \\
\text { Network Units }\end{array}$ & 16 \\
\hline $\mathrm{G}$ & Total bandwidth & $1 \mathrm{Gbps}$ \\
\hline $\mathrm{D}$ & OLT - ONU distance & $20 \mathrm{Km}$ \\
\hline $\mathrm{T}$ & Cycle time & $2 \mathrm{~ms}$ \\
\hline $\mathrm{T}_{\mathrm{g}}$ & Guard time & $1 \mu \mathrm{s}$ \\
\hline $\mathrm{G}_{\mathrm{max}}$ & $\begin{array}{c}\text { Initial maximum bandwidth } \\
\text { to be allocated to each ONU }\end{array}$ & $62.5 \mathrm{Mbps}$ \\
\hline $\mathrm{S}_{\text {voice }}$ & Limited bandwidth for voice & $64 \mathrm{kbps}$ \\
\hline $\mathrm{S}_{\text {video }}$ & Limited bandwidth for video & $4 \mathrm{Mbps}$ \\
\hline $\mathrm{S}_{\text {data }}$ & Limited bandwidth for data & $2 \mathrm{Mbps}$ \\
\hline
\end{tabular}

Bandwidth utilization is a parameter which measures the total bandwidth utilized of the total available bandwidth.

$$
\text { Bandwidth utilization }=\frac{\mathrm{B}_{\text {used }}^{\text {actual }}}{\mathrm{B}_{\text {offered }}^{\text {total }}} * 100 \%
$$

The comparative study is made to know how the bandwidth utilization is affected by the varying load. The graph for bandwidth utilization vs. offered load for three algorithms is shown in the Figure 2. The bandwidth utilization is high for the proposed UPCF algorithm compared to the other algorithms. To some extent the bandwidth utilization factor is same to the three algorithms when offered load values are lower.

The bandwidth utilization is maximum for the proposed UPCF algorithm compared to the existing DBA algorithms, Min DBA and RDM DBA when offered higher load values. The proposed UPCF algorithm utilizes bandwidth to the maximum extent thereby providing better QoS.

Second parameter considered for performance evaluation is the fairness index. The fairness index shows how far a fair allocation of bandwidth is done between the ONUs. The fairness index lies between zero and one $(0<\mathrm{F} \leq 1)$ and is proposed by R.P. Jain. It is given by the equation:

$$
F=\frac{\left(\sum_{i=1}^{N} B_{i}\right)^{2}}{N\left(\sum_{i=1}^{N} B_{i}^{2}\right)}
$$

Where $\mathrm{N}$ is the number of ONUs and $\mathrm{B}_{\mathrm{i}}$ is the bandwidth allocated to each $\mathrm{ONU}^{13}$. The simulated result is shown in the Figure 3. From the graph shown, we observe that the fair amount of bandwidth is allocated to each ONU by the proposed UPCF algorithm compared to other two algorithms.

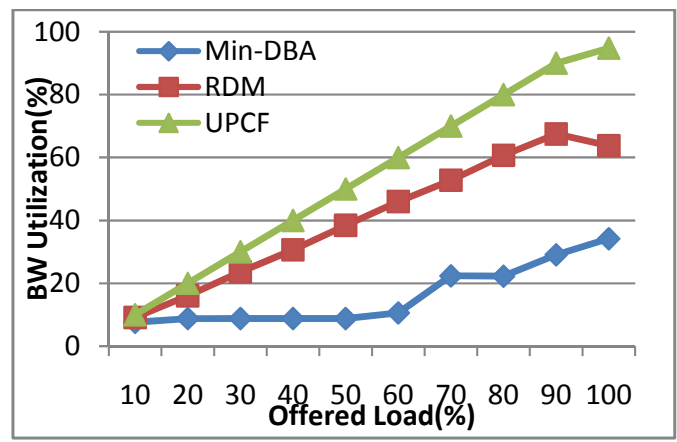

Figure 2. Bandwidth utilization. 


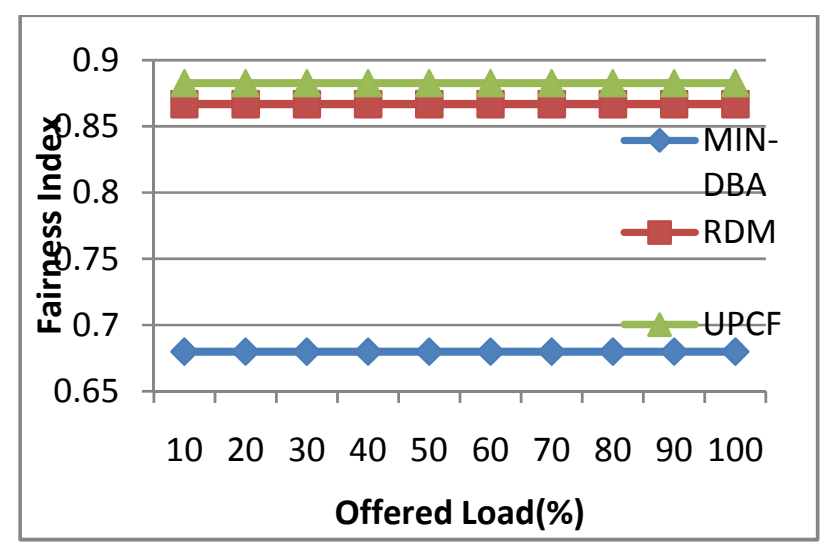

Figure 3. Fairness index.

The third parameter considered for the performance evaluation is delay analysis. Packet delay is expressed as the time elapsed between the packet arrival and the packet set off from the $\mathrm{ONU}^{14}$.

$$
\mathrm{d}=\mathrm{d}_{\text {poll }}+\mathrm{d}_{\text {grant }}+\mathrm{d}_{\text {queue }}
$$

Polling delay is expressed as the time elapsed between the arrival of packet in the ONU and the REPORT sent to the OLT, where on average ${ }^{14}$.

$$
\mathrm{d}_{\text {poll }}=\frac{\mathrm{T}_{\max }}{2}
$$

Where $\mathrm{T}_{\max }$ is the maximum cycle time.

Grant delay is the time duration between the request from ONU and the GATE message received from the OLT. The computation of $d_{\text {grant }}$ is shown in the equation given below ${ }^{14}$ :

$$
\mathrm{d}_{\text {grant }}=T\left[\frac{q-\mathrm{W}_{i . p}}{\mathrm{~W}_{\max }}\right]
$$

Where $\mathrm{q}$ is queue size and $\mathrm{W}_{\mathrm{i}, \mathrm{p}}$ is the pending queue size.

Queuing delay is insignificant as compared to polling delay and grant delay ${ }^{14}$.

$$
\mathrm{d}_{\text {queue }}=\left\{\begin{array}{cc}
\frac{q}{\mathrm{R}_{\mathrm{T}}}, & q<\mathrm{W}_{i, p} \\
\frac{\left(q-\mathrm{W}_{i . p}\right) \bmod _{\max }}{\mathrm{R}_{T}}, & q \geq \mathrm{W}_{i, p}
\end{array}\right.
$$

The delay for each traffic class is evaluated. The delay for voice traffic is shown in the Figure 4(a). From the graph we can arrive at a conclusion that the proposed
UPCF algorithm has lesser delay compared to Min DBA and RDM DBA.

The delay for the video traffic class is shown in the Figure 4(b). Depending on the varying offered load values, the delay is also changing. The delay for the data traffic class is shown in the Figure 4(c). The delay is lesser compared to other two algorithms since $\mathrm{W}_{\max }$ is higher in the proposed UPCF algorithm. The graphs for the allocated vs. requested bandwidth are plotted for each traffic class and are shown in Figure 5. UPCF algorithm shows a considerable amount of bandwidth

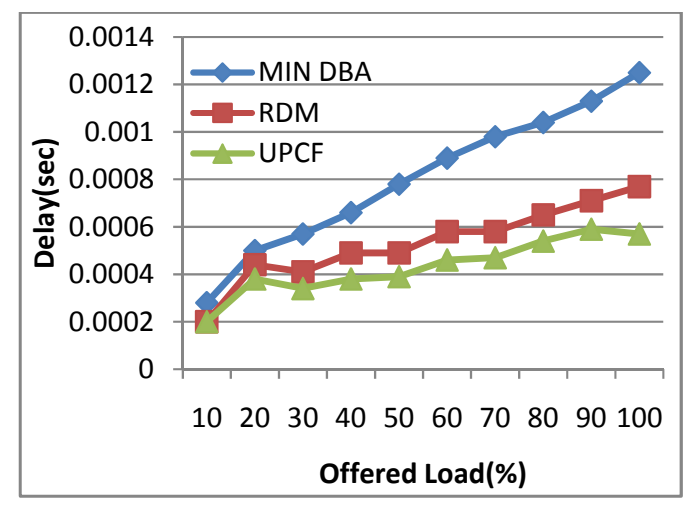

(a)

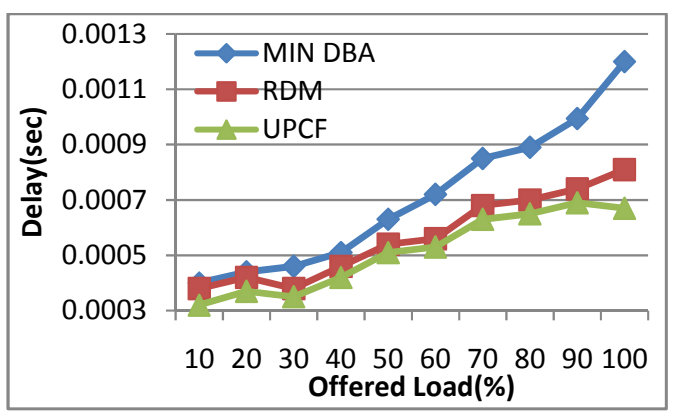

(b)

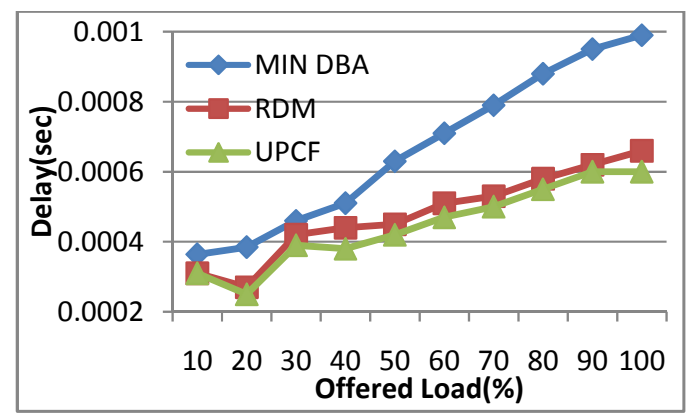

(c)

Figure 4. (a) Voice delay (b) Video delay (c) Data delay. 


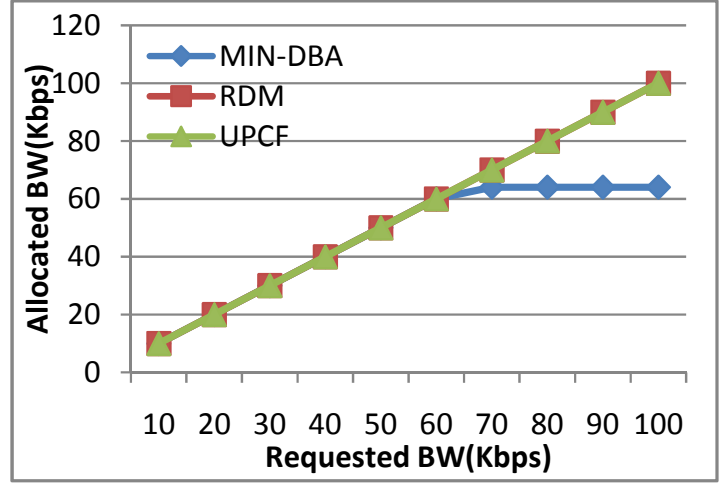

(a)

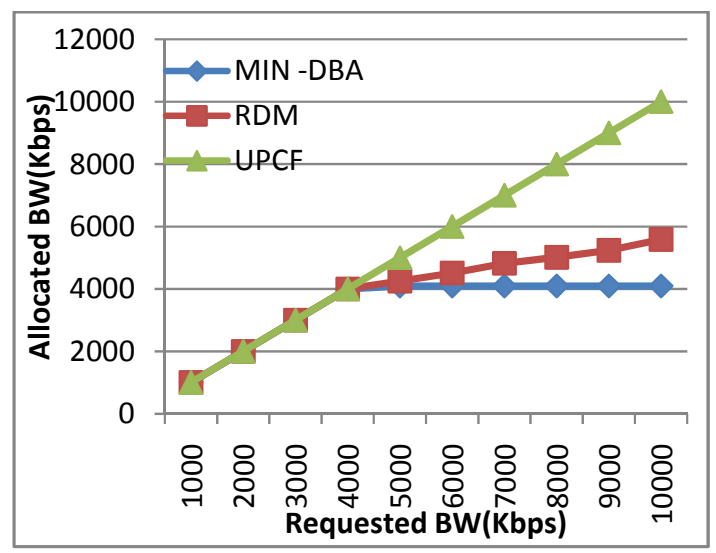

(b)

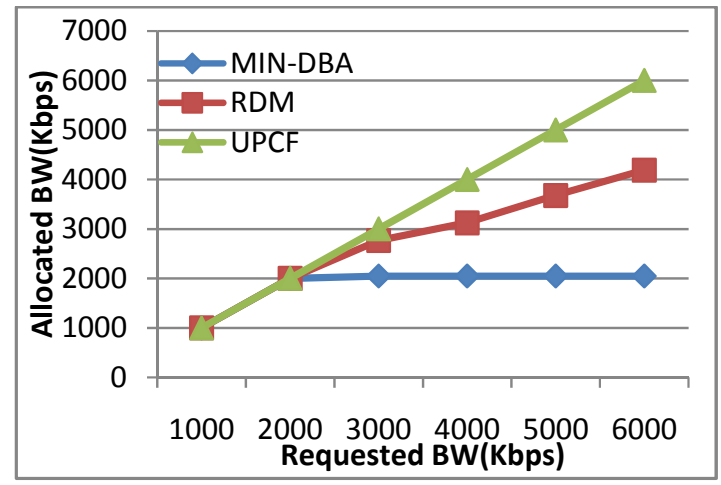

(c)

Figure 5. (a) For voice traffic (b) For video traffic (c) For data traffic.

utilization compared to other algorithms. From the results one can clearly understand that the proposed UPCF algorithm is better than the existing DBA algorithms in performance, bandwidth utilization, fairness index and packet delay.

\section{Conclusion}

The available bandwidth is used to the maximum extent in the proposed UPCF algorithm compared to other existing DBA algorithms, Min DBA and RDM DBA by increasing bandwidth utilization factor. The fairness of the proposed UPCF algorithm is higher compared with other algorithms as bandwidth is fairly divided among the ONUs. The packet delay is also less in the proposed algorithm.

\section{Acknowledgment}

The authors wish to acknowledge R. Arun Praveen, Mokirala K. Anjith and K. Charan Thej Reddy, Students of VIT University for implementing the algorithms in MATLAB.

\section{References}

1. YinY, Poo GS. User-oriented hierarchical bandwidth scheduling for Ethernet passive optical networks. Computer Communication. 2010 May; 33(8):965-75.

2. Byungjoo P, Ankyu H, Jae HY. Enhanced dynamic bandwidth allocation algorithm in Ethernet passive optical networks. ETRI Journal. 2008 Apr; 30(2):301-7.

3. IEEE 802.3ah Standards. Media access control parameters Physical layers and management parameters for subscriber access networks. IEEE Draft 802.3ah/D1.414; 2004 Sep.

4. BaiX, Shami A, AssiC. On the fairness of dynamic bandwidth allocation schemes in Ethernet passive optical networks. Computer Communication. 2006 Jul; 29(11):2123-35.

5. Kramer G, Mukherjee, B, Pesavento G. IPACT a dynamic protocol for an Ethernet PON (EPON). IEEE Commun Mag. 2002 Feb; 40(2):74-80.

6. Kramer G, Mukherjee B, Pesavento G. Ethernet PON (EPON): Design and analysis of an optical access network. Photonic Netw Commun. 2001 Jul; 3(3):307-19.

7. Kramer G, Mukherjee B, Dixit S, Ye Y, Hirth R. Supporting differentiated classes of service in Ethernet passive optical networks. J Opt Netw. 2002; 1(8):280-98.

8. Zahr SA, Gagnaire M. An analytical model of the IEEE 802.3ah MAC protocol for EPON-based access systems. Telecom Paris, 2006 May. p. 1-4.

9. Min L, Xiaomei F, Yu C, Fulei D. New dynamic bandwidth allocation algorithm for Ethernet PON. In: 8th Int conf on electronic measurement and instruments: 2007. 3 p. 224-7.

10. Sadon SK, Din NM, Al-Mansoori MH, Radzi NA, Mustafa IS, Yaacob I, Majid MSA. Dynamic hierarchical 
bandwidth allocation using Russian Doll Model in EPON. Computers and Electrical Engineering. 2012 Nov; 38(6):1480-9.

11. Assi C, Ye Y, Dixit S, Ali M. Dynamic bandwidth allocation for quality-of-service over Ethernet PONs. IEEE Journal of Selected Areas in Communication. 2003 Nov; 21(9):1467-77.

12. Bai X, Shami A, Assi C. On the fairness of dynamic bandwidth allocation schemes in Ethernet passive optical networks. Comput Commun. 2006 Jul; 29(11):2123-35.
13. Jain R, Durressi A, Babic G. Throughput fairness index: An explanation. ATM Forum Contribution; 1999. Available from: http:/www.cse.wustl.edu/ jain/atmf/ftp/ af_fair.pdf

14. Kramer G, Mukherjee B, Pesavento G. Interleaved polling with adaptive cycle time (IPACT): A dynamic bandwidth distribution scheme in an optical access network. Photonic Netw Commun. 2002 Jan; 4(1):89-107. 